\title{
Social media: physicians-to-physicians education and communication
}

\author{
Keith A. Fehring ${ }^{1} \cdot$ Ivan De Martino ${ }^{2}$ - Alexander S. McLawhorn ${ }^{3} \cdot$ Peter K. Sculco $^{3}$
}

Published online: 24 March 2017

(C) Springer Science+Business Media New York 2017

\begin{abstract}
Purpose of review Physician to physician communication is essential for the transfer of ideas, surgical experience, and education. Social networks and online video educational contents have grown exponentially in recent years changing the interaction among physicians.

Recent findings Social media platforms can improve physician-to-physician communication mostly through video education and social networking. There are several online video platforms for orthopedic surgery with educational content on diagnosis, treatment, outcomes, and surgical technique. Social networking instead is mostly centered on sharing of data, discussion of confidential topics, and job seeking. Quality of educational contents and data confidentiality represent the major drawbacks of these platforms.

Summary Orthopedic surgeons must be aware that the quality of the videos should be better controlled and regulated to avoid inaccurate information that may have a significant impact especially on trainees that are more prone to use this type of resources. Sharing of data and discussion of confidential
\end{abstract}

This article is part of the Topical Collection on Social Media and Orthopedics

Keith A. Fehring

keith.fehring@orthocarolina.com

1 Hip \& Knee Center, OrthoCarolina, 2001 Vail Avenue Suite 200A, Charlotte, NC 28207, USA

2 Complex Joint Reconstruction Center, Department of Orthopaedic Surgery, Hospital for Special Surgery, 535 East 70th Street, New York, NY 10021, USA

3 Adult Reconstruction and Joint Replacement Division, Department of Orthopaedic Surgery, Hospital for Special Surgery, 535 East 70th Street, New York, NY 10021, USA topics should be extremely secure according the HIPAA regulations in order to protect patients' confidentiality.

Keywords Social media $\cdot$ Social networking $\cdot$ Educational video $\cdot$ Internet $\cdot$ Physician-physician relations

\section{Introduction}

Orthopedic surgeons can use collaborative social media programs, tools, and video for communication, teaching, and case discussion. These programs allow for surgeons to be up-todate on new technology and communicate regarding different advances or techniques in the field. These P2P (Physician-toPhysician) outlets can be divided into video teaching [i.e., VuMedi (http://www.vumedi.com), OR Live (http://www. orlive.com)], online social networks [i.e., Orthogate (http:// www.orthogate.org)], and case discussion boards [i.e., Orthogate, DocSpera (http://www.docspera.com)]. These forums allow for orthopedic surgeons to communicate with their colleagues to share new ideas and techniques, discuss difficult cases, and discuss policies pertinent to the field of orthopedics. These new outlets have opened doors of communication through the use of the internet to connect surgeons from around the world. This section will review some of the available educational content online for orthopedic surgeons and also review the online research communities that have grown in recent years.

\section{Video teaching}

VuMedi and OR live have become valuable teaching tools for training surgeons to learn specific techniques through videos and lectures on various procedures. These sites provide hours 
of valuable video meant to teach those in training or learning new procedures. Intra-operative videos allow surgeons to watch these techniques multiple times to familiarize themselves with the operation prior to embarking on the procedure themselves. This does not take the place of formal orthopedic mentorship training, but rather provides another outlet for learning in an ever-evolving field. The use of these educational sites meant for surgeons to educate surgeons have shown great promise during a time when the 80 -h-work week for residency training is being looked at with increased scrutiny. These video outlets allow training surgeons to learn while not being "on the job." One limitation of these platforms is their lack of peer review. These outlets allow for any surgeon to post videos which bring inherent bias and risk involved with possibly teaching techniques that are not ideal for all orthopedic surgeons [1]. Webinars regarding the management of difficult orthopedic problems have become another source of communication among surgeons. These webinars provide lectures, videos, and communication among surgeons teaching and those attending the webinar. It provides an interactive environment for communication about various orthopedic problems.

There are several online video platforms for orthopedic surgery, with educational content on diagnosis, treatment, outcomes, and surgical technique developed specifically for orthopedic surgeons and sub-specialists. VuMedi advertises itself as the "YouTube" for specialist physicians. This platform is a surgeon-only video sharing website where surgeons can view, upload, and discuss surgical videos. This free video education platform for physicians, fellows, and residents has an excellent variety of videos on a variety of topics. There is also community of physicians who can post comments, and the surgeons can then respond to these threads. However, videos are not peer reviewed and may teach unverified techniques.

The American Academy of Orthopedic Surgeons (AAOS) has an online orthopedic video program, the "Orthopedic Video Theater" (www.aaos.org/education/ovt/) which provides continuously updated video contents for orthopedic surgeons from renowned experts in each subspecialty. Videos are peer reviewed, but a subscription fee is required to have access to this orthopedic video program.

The Hospital for Special Surgery eAcademy (www.hss. classroom 24-7.com) is an electronic, digital platform for distribution of education and academic programs through live streaming as well as offerings on demand. This hospital-driven-platform provides academic programs, lectures, and courses and includes live streaming, videos, webcasts, and webinars on demand. The material provided is peer reviewed, and a registration is needed to access the contents of this platform.

AO Surgery Reference (www.aosurgery.org) is a website created by the AO (Arbeitsgemeinschaft für Osteosynthesefragen) Foundation and represents a comprehensive guide for management of fractures. This website is focused on fracture management by providing the most recent peer-reviewed-clinical principles as they apply to the diagnosis, preparation, execution, and aftercare of orthopedic trauma. This website is free and no registration is needed [2].

\section{Online social networks}

OrthoGate has led the way as the largest forum for communication about prospective orthopedic residents and current residents on various topics pertaining to residency training. These forums allow for the most up-to-date information regarding the application process, various programs, and news from the Accreditation Council for Graduate Medical Education (ACGME). As stated on their website, their mission is "to improve orthopaedic care, education, and research through internet technologies." These forums strive to facilitate access for orthopedic professionals to information and to each other for communication. OrthoGate has led the way through internet discussion boards regarding various topics in orthopedics, but have also facilitated case discussion through their website. The online forums allow for users to post their questions about difficult cases and allow other surgeons to respond with advices, experience, and techniques regarding a specific case.

DocSpera is an online community that connects surgeons from around the world to discuss difficult cases and is an exclusive partner of the American Academy of Hip and Knee Surgeons (AAHKS). It allows for the easy uploading of case details as well as images to help facilitate communication regarding the case. AAHKS members help proctor the case discussions and facilitate problem solving with each individual case. It is a secure and HIPAA compliant platform that allow confident case sharing among surgeons.

\section{Academic research: sharing and collaboration}

ResearchGate (www.researchgate.net) is a social networking site for researchers and scientists to share papers, ask and answer questions, and find collaborators. Members of this social network have a user profile and can upload papers, research proposals, and presentations. The researchers can interact with other researchers following their activities and engaging in discussions with them. Scientists can share data, discuss confidential topics, and seek for a job. The New York Times described the site as a mashup of Facebook, Twitter, and LinkedIn [3].

Academia.edu (www.academia.edu) is another social networking site for researchers and scientists. This website seems to reflect a combination of social networking norms and academic norms. This platform is mostly used to increase the viewership and number of citations of published research instead of favoring discussion among researchers. 


\section{Physician to physician: job listings}

While social networks like Facebook and Twitter are great for engaging patients and attracting new business, the benefits of LinkedIn (www.linkedin.com) revolve more around professional development.

Some social media consultants recommend that physicians begin their social medial professional presence with activating a LinkedIn profile. A LinkedIn profile takes about $45 \mathrm{~min}$ to create and is essentially a digital translation of your CV. Benefits to developing a LinkedIn profile include the ability for professional acquaintances to locate the professional CV online and get a better understanding of professional career and credentials. With LinkedIn's professional reputation, its use among doctors has been increasing.

Doximity (www.doximity.com) has been called "the LinkedIn for doctors" and has reportedly signed up more than half of all physicians in the USA in 2015 [4]. Doximity is also a social and professional network for doctors. The Doximity platform differentiates itself from LinkedIn by recognizing the need for a secure platform where physicians can communicate with one another. Doximity hopes to modernize and improve communication between health care professionals. Doximity is safe and HIPAA secure in order to protect patients' confidentiality during case sharing among doctors.

\section{Conclusion}

Social media platforms offer the possibility for orthopedic surgeons to communicate among them. These platforms can help physicians to transfer ideas, surgical experience, and education and look for job listings. In this way, education and case discussion can happen anytime and not restricted to work hours. However, the quality of the videos should be better controlled and regulated to avoid inaccurate information that may have a significant impact especially on trainees that are more prone to use this type of resources. Communication among doctors should be extremely secure according the HIPAA regulations in order to protect patients' confidentiality. Sharing of data, discussion of confidential topics, and job seeking are additional opportunities for surgeons that can happen through these social media platforms.

\section{Compliance with ethical standards}

Conflict of interest All authors declare that they have no conflict of interest.

Human and animal rights and informed consent This article does not contain any studies with human or animal subjects performed by any of the authors.

\section{References}

1. Urch E, Taylor SA, Cody E, Fabricant PD, Burket JC, O'Brien SJ, Dines DM, Dines JS. The quality of open-access video-based orthopaedic instructional content for the shoulder physical exam is inconsistent. HSS J. 2016 Oct;12(3):209-15.

2. Lin T. Cracking Open the Scientific Process. The New York Times, Page D1 of the New York ed. 17 Jan 2012.

3. Nambiar M, West LR, Bingham R. AO surgery reference: a comprehensive guide for management of fractures. Br J Sports Med. 2016; doi:10.1136/bjsports-2016-096677.

4. http://venturebeat.com/2014/12/09/doximity-the-linkedin-fordoctors-has-signed-up-more-than-half-of-u-s-physicians/ 